\title{
Otoprotection Mechanisms Against Oxidative Stress Caused by Cisplatin
}

\author{
Maiara Santos Gonçalves ${ }^{1}$ Aron Ferreira da Silveira ${ }^{2}$ Adriana de Andrade Batista Murashima ${ }^{3}$ \\ Maria Rossato ${ }^{3}$ Miguel Angelo Hippolito ${ }^{3}$
}

1 Department of Phonoaudiology, Universidade Federal de Santa Maria, Santa Maria, RS, Brazil

2 Department of Morphology, Universidade Federal de Santa Maria, Santa Maria, RS, Brazil

${ }^{3}$ Department of Ophthalmology, Otorhinolaryngology and Head and Neck Surgery, Faculdade de Medicina de Ribeirão Preto da Universidade de São Paulo, Ribeirao Preto, SP, Brazil
Address for correspondence Maiara Santos Gonçalves, Departamento de Fonoaudiologia, Universidade Federal de Santa Maria, Avenida Roraima, 1000, Santa Maria, RS, 97105900, Brazil (e-mail: maiarasg@yahoo.com.br).

Int Arch Otorhinolaryngol 2020;24:e47-e52.

\begin{abstract}
Keywords

- cisplatin

- toxicity

- free radicals

- cochlea

- oxidative stress

Introduction Cisplatin damages the auditory system and is related to the generation of free radicals. Glutathione peroxidase is an endogenous free radicals remover.

Objective To investigate the mechanisms involved in otoprotection by $\mathrm{N}$-acetylcysteine through the expression of glutathione peroxidase in outer hair cells from rats treated with cisplatin.

Methods Male Wistar rats were intraperitoneally injected with cisplatin $(8 \mathrm{mg} / \mathrm{Kg})$ and/or received oral administration by gavage of $\mathrm{N}$-acetylcysteine $(300 \mathrm{mg} / \mathrm{Kg})$ for 3 consecutive days. On the $4^{\text {th }}$ day, the animals were euthanized and beheaded. The tympanic bullae were removed and prepared for scanning electron microscopy and immunofluorescence.

Results Among the groups exposed to ototoxic doses of cisplatin, there was an increase in glutathione peroxidase immunostaining in two groups, the one exposed to cisplatin alone, and the group exposed to both cisplatin and $\mathrm{N}$-acetylcysteine.

Conclusion The expression of glutathione peroxidase in the outer hair cells of rats exposed to cisplatin showed the synthesis of this enzyme under cellular toxicity conditions.
\end{abstract}

\section{Introduction}

The cisplatin-based regimen is considered the gold standard for the treatment of a variety of solid tumors. ${ }^{1}$ This antineoplastic agent acts in the cell by reducing the levels of endogenous antioxidant enzymes and activating the apoptotic cascade. ${ }^{2,3}$ Despite its proven efficacy, cisplatin should be administered with caution due to its side effects, which include nephrotoxicity, neurotoxicity, and ototoxicity. ${ }^{4}$

There are different mechanisms through which cisplatin causes injury and awakens the cell death pathway in the auditory system. The mechanism most discussed currently relates to the generation of free radicals, specifically the reactive oxygen species (ROS), which cause injuries and can lead to cell death by apoptosis. ${ }^{5}$

Damages to the auditory system occur with both acute high doses and cumulative doses. The hearing loss is bilateral sensorineural, irreversible, and associated with tinnitus. ${ }^{5}$ Due to the tonotopic arrangement of the cochlea, high frequencies are affected first, ${ }^{5,6}$ since damages to the outer hair cells (OHCs) progress from the base to the cochlea apex and from the third to the first row of these cells; then, the damage affects the inner hair cells (IHCs). ${ }^{7}$ Still, the injuries are not limited to hair cells, they also affect supporting cells, the stria vascularis, and the spiral ganglion. ${ }^{8}$ received

July 4, 2019

accepted

September 7, 2019
DOI https://doi.org/

10.1055/s-0039-1698782. ISSN $1809-9777$.
Copyright ( $\odot 2020$ by Thieme Revinter

Publicações Ltda, Rio de Janeiro, Brazil
License terms

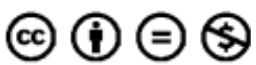


In the cochlea, cisplatin accumulates in the tissue, integrates into the DNA and causes disorder in protein synthesis and antioxidant enzymes. As the cochlea is in an isolated anatomical position and is virtually a closed system, it becomes unable to expel the accumulated toxin at the same rate and speed at which it is generated. Thus, there is a ROS overload associated with an impaired antioxidant system. ${ }^{9}$ This condition causes lipid peroxidation increase, thus initiating events that culminate in hair cells, supporting cells, stria vascularis, and auditory nerve apoptosis. ${ }^{10}$

Facing irreversible ototoxicity, the challenge has been to discover and enable a substance with otoprotective action that does not interfere with the cytotoxic effect of cisplatin in tumor cells. Among these substances is N-acetylcysteine (NAC), a drug from the group of thiols with potential antioxidant effect that increases the levels of intracellular glutathione. ${ }^{11,12}$ Clinically, NAC is used as a mucolytic agent to clear air pathways and as an antidote to acetaminophen poisoning. ${ }^{13}$ This compound was selected for the study protocol by presenting antioxidant properties, having a free radicals direct scan action and an indirect action, serving as a precursor of intracellular free cysteine, which can be used to synthesize glutathione peroxidase. Glutathione peroxidase (GSH-Px) is an endogenous free radicals remover, ${ }^{14}$ and it is present in the cell cytoplasm and in the mitochondria. ${ }^{15}$

Thus, this study aimed to investigate the mechanisms involved in otoprotection by NAC through the expression of GSH-Px in the OHCs from rats treated with cisplatin.

\section{Methods}

The study population consisted of male Wistar rats subjected to a light-dark cycle of $12 / 12$ hours (light period started at 7 AM), kept at room temperature $\left(19-23^{\circ} \mathrm{C}\right)$ and humidity $(55 \% \pm 15 \%)$ controlled and also with water and food ad libitum. All procedures were performed according to the criteria of the Arouca Law (Law $n^{\circ} 11.794$, of October 8, 2008) and the Brazilian Society of Laboratory Animal Science (Sociedade Brasileira de Ciências em Animais de Laboratório). The project was submitted for approval by the Ethics Committee on Animal Experimentation of the institution of origin.

Animals weighing between 260 and $300 \mathrm{~g}$, with Preyer ${ }^{16}$ reflex present and electrophysiological threshold of brainstem auditory evoked potential (BAEP) of $20 \mathrm{~dB} \mathrm{HL}$ in both ears were included in the study.

Animals that showed signs of otitis external or acute otitis media at examination of the external auditory canal, , those that had earwax that was difficult to remove, and those with very narrow canals that prevented placing the probe for BAEP testing were excluded from the sample.

The drugs administered were cisplatin (Tecnoplatin - Eurofarma Laboratórios Ltda, São Paulo, SP, Brazil); acetyl-cysteine (Laboratório EMS, Santo André, SP, Brazil); ketamine hydrochloride 10\% (Ketamin 50mg/ml - Laboratório Cristália, Itapira, SP, Brazil); xylazine hydrochloride (Dopaser 200mg - Laboratório Hertape Calier, Juatuba, MG, Brazil).

For immunofluorescence, Rabbit polyclonal antibody and anti- GSH-Px 1 (100 $\mu \mathrm{g}$ presentation) were used; goat polyclonal secondary antibody anti-IgG - H\&L ( Alexa Fluor 488-Thermo Fisher Scientific, Waltham, MS, USA); goat polyclonal secondary antibody anti-IgG - H\&L (Alexa Fluor 594-Thermo Fisher Scientific).

Once the animals were selected according to the weight and the presence of Preyer reflex, they were kept in auditory rest for 24 hours, after which they were subjected to anesthesia and analgesia intramuscularly, as well as otoscopy and BAEP test. The following four groups were outlined randomly:

Group 1: negative control ( 3 animals - 6 cochleae), administration of $0.9 \%$ saline solution intraperitoneally, at the same volume corresponding to the dose of cisplatin for 3 consecutive days;

Group 2: positive control ( 3 animals -6 cochleae), oral administration by gavage of $300 \mathrm{mg} / \mathrm{Kg} /$ day of $\mathrm{N}$-acetylcysteine for 3 consecutive days;

Group 3: ototoxic ( 5 animals - 10 cochleae), intraperitoneal administration of $8 \mathrm{mg} / \mathrm{Kg} /$ day of cisplatin for 3 consecutive days;

Group 4: ototoxic with otoprotection (5 animals - 10 cochleae), oral administration by gavage of $300 \mathrm{mg} / \mathrm{Kg} / \mathrm{day}$ of $\mathrm{N}$-acetylcysteine, 1 hour before the intraperitoneal administration of $8 \mathrm{mg} / \mathrm{Kg} /$ day of cisplatin for 3 consecutive days.

On the $4^{\text {th }}$ day, 24 hours after the last drugs and saline solution administration, the animals were anesthetized again and received analgesics. They underwent otoscopy to rule out those with external and/or middle ear disease. Immediately afterwards, the animals were euthanized with a lethal dose of Tiopental (Abbott Laboratórios do Brasil, São Paulo, SP, Brasil) and beheaded. The tympanic bullae were removed and prepared for scanning electron microscopy (SEM) and immunofluorescence.

\section{Procedures}

\section{Brainstem Auditory Evoked Potential}

The electrophysiological threshold was obtained by BAEP with the Smart EP 3.86USBez (Intelligent Hearing Systems, Miami, FL, USA) equipment. The acquisition was ipsilateral, with analysis time of 12 milliseconds and 1,000 promediation and replication sample. The stimulus was the click of 27.7/s issued by model Ear Tone 3A insert earphones (Vitasons, Porto Alegre, Rio Grande do Sul, Brasil), in alternating polarity. The stimuli started at $90 \mathrm{~dB}$ HL with gradual reduction to the lowest stimulus intensity to highlight wave II. ${ }^{17}$

\section{Histological Preparation}

The tympanic bullae were removed from the cephalic segment with dissection scissors posteriorly placed at the cervical spine, making a longitudinal median section on the skull. The bullae were located manually, with the external auditory meatus as a guide, and removed, thus exposing the cochleae. Having an optical microscope as an auxiliary tool, the cochleae had their oval and round windows broken and their apex drilled for fixing solution of $3 \%$ glutaraldehyde was injected; the cochleae were kept in this solution at $4^{\circ} \mathrm{C}$ for $24 \mathrm{~h}$. Afterwards, the cochleae received phosphate buffered baths for cell cleaning and underwent microdissection. Later on, the samples were 
decalcified in a solution of $8 \%$ ethylene diamine tetraacetic acid (EDTA) (Merck Laboratory, Darmstadt, Germany), $0.55 \mathrm{~g}$ of $\mathrm{NaOH}$ (Merck Laboratory) and $100 \mathrm{ml}$ of distilled water. After decalcification, the material was dehydrated in increasing concentrations of ethanol (50\%, 70\%, 80\%, 90\%, 95\%, and absolute ethanol) and received 320 -minute baths of $100 \%$ ethanol, allowing the structures to be immersed, in the last bath, at room temperature for 12 hours. Once dehydrated, the material was submitted to diaphanization with xylene baths. Then, to completely remove the xylol and facilitate paraffin infiltration, the material was passed through 2 changes of molten paraffin at the temperature of $60^{\circ} \mathrm{C}$. After that, the inclusion in paraffin was conducted in an oriented manner, and the cochleae were positioned to be sectioned at microtome longitudinally, parallel to the modiolus, at a thickness of 6 microns.

After obtaining the tissue serial sections in depth modiolus (at least three sections of each cochlea), those serial sections were arranged on a glass slide with distilled water on its surface. Then, the slide was placed in heated platinum for the tissue to be stretched, after which it was put in an oven heated to $60^{\circ} \mathrm{C}$. After that, the slides were subjected to immunofluorescence protocol.

\section{Immunofluorescence}

The slides preparation for immunofluorescence conformed to the following protocol: 1) two xylene 10-minute baths for eliminating paraffin; 2) hydration with decreasing concentrations of ethanol (absolute alcohol, 95\%, 70\%, 50\%) and distilled water; 3) immersion in pH6 citrate buffer for 1 hour in a water bath; 4) three distilled water 5-minute baths; 5 ) a phosphate-buffered saline (PBS) and glycine 30minute bath; 6 ) bovine serum albumin (BSA $2 \%$ ) and Tween20 1-hour blocking; 7) overnight primary antibody incubation in a dark and humid chamber; 8) five phosphate buffered saline (PBS), with light protected blades, 5-minute baths; 9 ) one hour incubation with secondary antibody in a dark and humid chamber;10) five PBS, with light protected blades, 5-minute baths. Then, the slides were covered with a glass cover and stored in refrigerator. The confocal microscope analysis was initiated on the next day with the Leica SP5 DM4000 B Led and |Leica LAS - AF Lite software (Leica Microsystems, Wetzlar, Alemanha).

\section{Scanning Electron Microscopy (SEM)}

For the anatomical analysis of the organ of Corti, one rat cochlea from each group was prepared to be analyzed at a SEM. The samples were dissected, partially prepared, and set by the base of a cylindrical metal specimen holder with conductive carbon paste. The structures were then covered by a thin layer of 24 carat gold through BAL-TEC - SCD 050 Sputter Coater (Bal-Tec AG, Balzers, Liechtenstein) evaporation process, making it electrically conductive. When the structures preparation processes were completed, the cochleae were analyzed with JEOL Scanning Electron Microscope - JSM 5200 (JEOL Ltd., Akishima, Tokyo, Japan).

The OHC integrity or injury condition was defined by their stereocilia analysis. Cells with stereociliae perfectly shaped and arranged were considered healthy (normal). ${ }^{18,19}$

\section{Results}

\section{Anatomical Evaluation by SEM}

As rats have two and half turns in the cochlea, the middle third of the basal turn and of the second turn (or medium turn) was considered as an anatomical analysis parameter. The apical turn was excluded for presenting a natural breakdown of OHC stereocilia ' $w$ ' pattern, thus hindering the structural analysis.

In the -Fig. 1 grouping, it is possible to observe $\mathrm{OHC}$ anatomical integrity in all groups, evidenced by maintenance of their stereocilia "w" pattern.

\section{Glutathione Peroxidase Expression Evaluation through Immunofluorescence}

The GSH-Px enzyme was developed using secondary antibody conjugated to Alexa Fluor 488 (Thermo Fisher Scientific); therefore, immunostaining occurred in green coloration. The blue fluorescence indicates cell nuclei labeled with 4',6diamidino-2-phenylindole (DAPI) dye.

Group 1 (negative control) - This Group was the comparison parameter to the other groups. Thus, the evaluation of GSH-Px expression in groups 2, 3, and 4, was determined by increasing the intensity of green coloring throughout the cell cytoplasm, and this expression was classified as granular.

During the confocal microscopy cochlear tissue longitudinal section analysis, the middle turn (C2) was selected for better visualization of the organ of Corti, since it proved to be structurally more preserved to mechanical artifacts inherent to histological preparation.

On the images in - Fig. 2, GSH-Px immunostaining is observed in groups 2, 3, and 4, indicated by the granular green staining in the cell cytoplasm.

\section{Discussion}

One of the mechanisms through which cisplatin damages the auditory system and awakens the apoptotic cascade is related to the generation of free radicals intracellularly. ${ }^{5}$ To combat reactive oxygen species (ROS) and keep the oxidative system balanced, cells have an endogenous antioxidant system. In the cochlea, this system includes glutathione, and enzymes superoxide dismutase (SOD), catalase (CT), GSH-Px, and glutathione reductase (GSH-R). ${ }^{9}$

Once generated and not controlled by the antioxidant system, ROS can react with a variety of cellular components, causing changes such as lipid aldehyde membrane peroxidation, proteins oxidative modification, and DNA lesions. ${ }^{15,20}$

Exogenous otoprotectors, including thiol-containing antioxidants (compounds containing sulfur), act at an earlier stage "sweeping" free radicals and preventing the cell death pathway onset, such as NAC. ${ }^{21}$ Rybak et al ${ }^{12}$ indicate that the high affinity of sulfur to platinum is the basis for thiol-containing compounds are widely used in research involving cisplatin toxicity.

Since the study by Feghali et $\mathrm{al}^{11}$, the otoprotective effect of NAC has been observed in hair cells and cochlear ganglion neurons (in vitro). Other studies on cell culture have found 

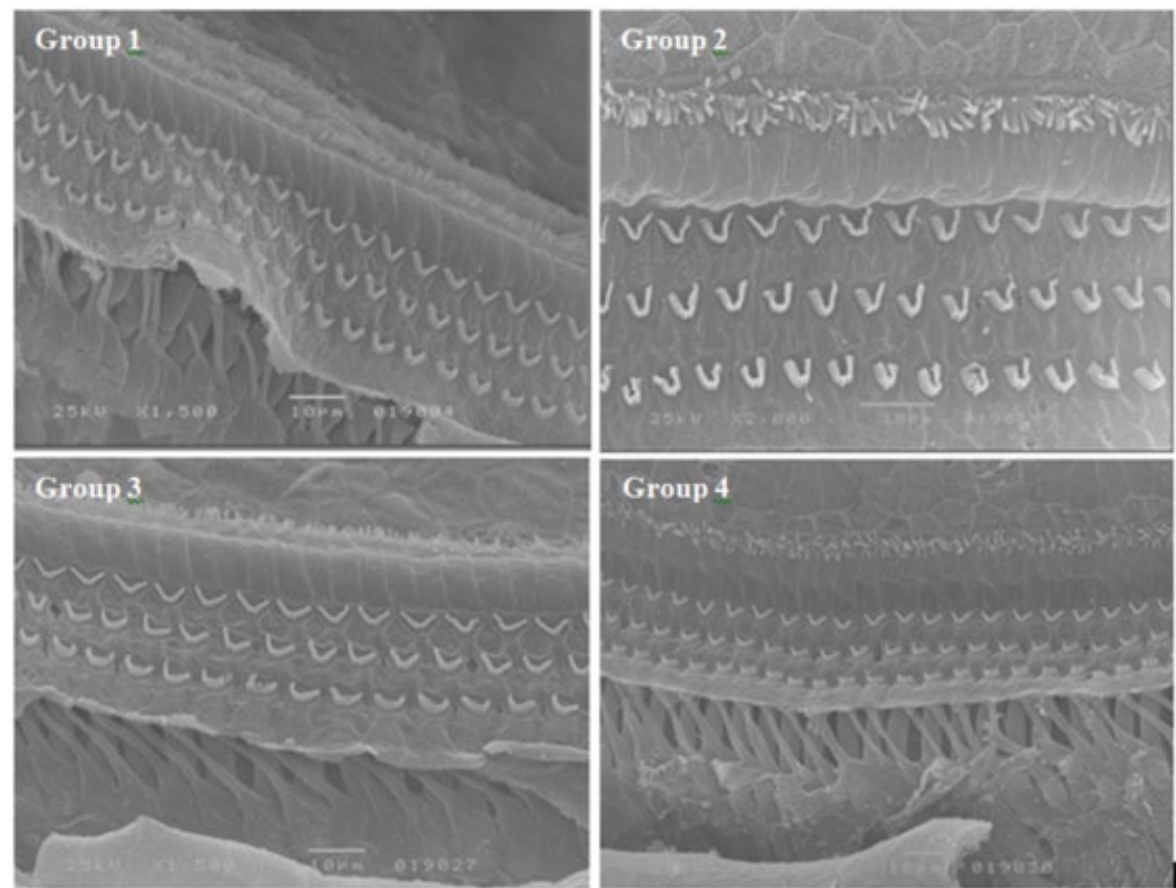

Fig. 1 Photomicrograph of the organ of Corti of rats in the four groups studied, showing the middle third of the basal whorl. The maintenance of ciliary architecture in the three rows of outer hair cells is observed in the four groups. 1,500x increase (groups 1 and 3); 2,000x increase (group 2); $1,000 x$ increase (group 4).
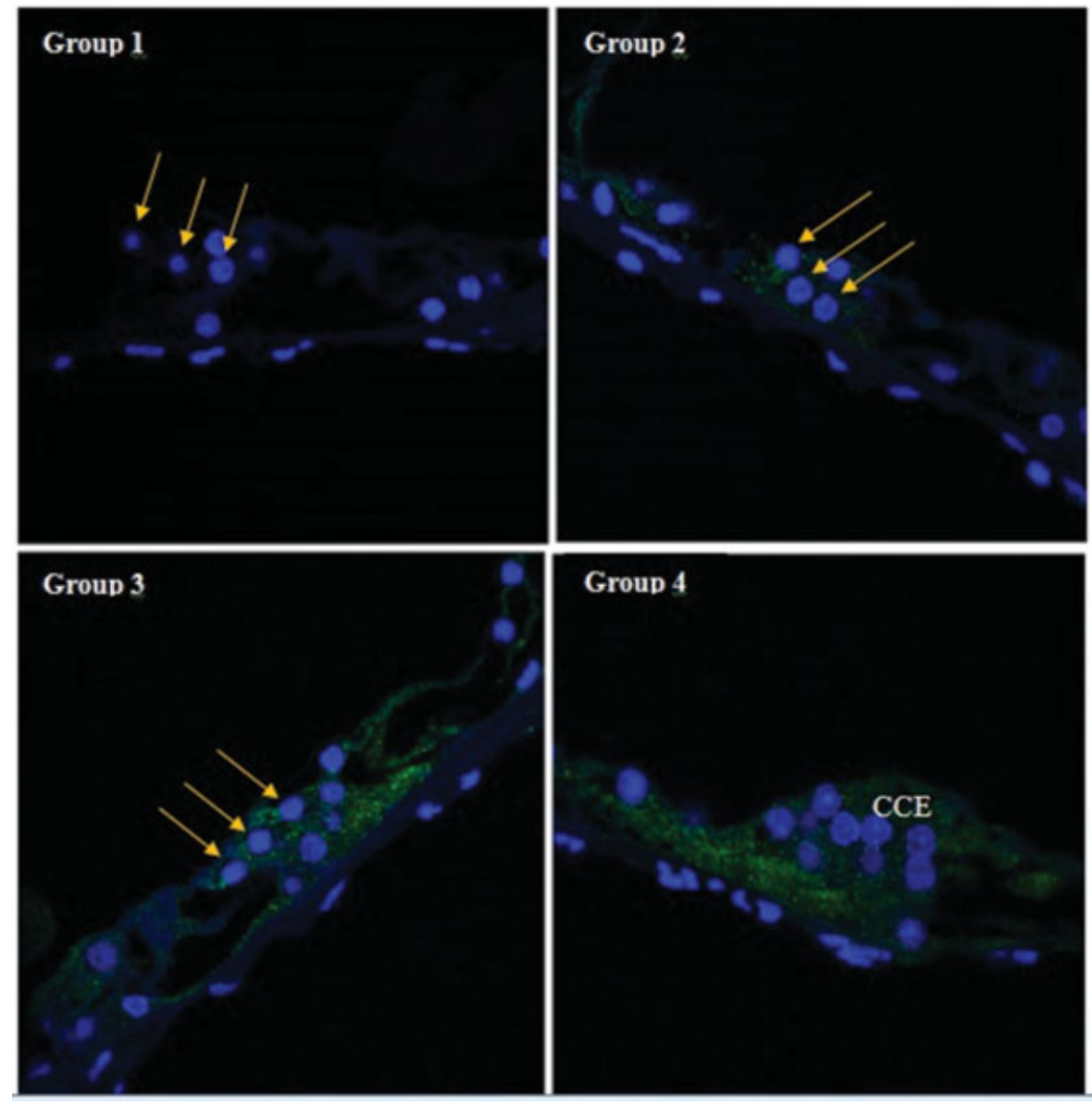

Fig. 2 Photomicrograph of the organ of Corti of rats in the four groups studied, regarding the immunostaining of glutathione peroxidase. Images overlay: cell nuclei labeled with 4',6-diamidino-2-phenylindole (blue) and immunostained cells with anti-glutathione peroxidase revealed with Alexa 488 (green). Arrows indicating the nucleus of outer hair cells(objective 63x, zoom 2.0). 
that NAC inhibited cisplatin-induced apoptosis when it occurred through the caspases ${ }^{22}$ signaling pathway, and, in vivo, it protected against ototoxicity and nephrotoxicity. ${ }^{6}$

Some otoprotectors are incompatible with cisplatin once they reduce its antineoplastic potential. However, studies show that this is not the case with NAC. Dickey et $\mathrm{al}^{6}$ suggest that the chemotherapy and otoprotection administration route and timing must be different in an attempt to keep the antitumor efficacy. Therefore, in this study, NAC was administered by gavage 1 hour before intraperitoneal cisplatin application.

From the method employed in this study, which sought to investigate the relationship between GSH-Px expression in the OHCs of rats under the effect of NAC and cisplatin, it was possible to verify the absence of immunostaining of this enzyme in the control group (-Fig. 2, group 1) on cellular integrity condition. In group 2 , in which the animals received $300 \mathrm{mg} / \mathrm{kg} /$ day of NAC and, thus, reached a cell integrity condition, on a qualitative assessment, there was a mild immunostaining for GSH-Px in all samples analyzed, demonstrating the ability of NAC in motivating their synthesis.

In the groups exposed to ototoxic doses of cisplatin, there was a significant increase in GSH-Px immunostaining both in the group exposed to cisplatin alone (-Fig. 2, group 3) and in the group exposed to both cisplatin and NAC (-Fig. 2, group 4). With this result, we can infer that, even without the presence of NAC, the cell has the capacity to react to the toxicity of cisplatin by raising the level of GSH-Px, which is one of the enzymes belonging to their endogenous antioxidant system.

Within the four groups studied in the present qualitative analysis, it appears that there was an increase in GSH-PX immunostaining in groups 2,3 , and 4 compared with group 1. However, between groups 3 and 4 , ototoxic and ototoxic with otoprotection, respectively, it was not possible to verify the spare difference in immunostaining.

Clinically, in rats, pretreatment with $400 \mathrm{mg} / \mathrm{kg}$ of intravenous NAC, 15 and 30 minutes before administration of $6 \mathrm{mg} / \mathrm{kg}$ cisplatin, intra-arterially, prevented ototoxicity, a situation that was confirmed by the BAEP thresholds. It was also observed that NAC prevented the weight loss caused by cisplatin, possibly by reducing the gastrointestinal tract toxicity. ${ }^{23}$ In addition, a more favorable status was observed in this study in the animals treated with NAC (group 4), with less weight and hair loss, compared with animals exposed to cisplatin alone (group 3).

The extent and degree of histological and functional changes in the auditory system are related to the dose of cisplatin administered. ${ }^{11}$ In this study, $8 \mathrm{mg} / \mathrm{kg} /$ day of cisplatin, representing a $24 \mathrm{mg} / \mathrm{kg}$ cumulative dose, was not toxic to the point where there would be noticeable anatomical changes in the OHCs observed through SEM (-Fig. 1). However, the same dose was able to induce an intrinsic cellular response to cisplatin toxicity, irrespective of stimulation by NAC, since there was significant increase in the immunolabeling of GSH-Px in group 3. It was not possible to quantify this expression, but qualitatively, in the opinion of two independent examiners, the expression that occurred in groups 3 and 4 did not differ among themselves.

\section{Conclusion}

The expression of GSH-Px in the OHCs of rats exposed to cisplatin showed the synthesis of this enzyme under cellular toxicity conditions. Furthermore, the OHCs react in the presence of cisplatin raising levels of GSH-Px, regardless of exogenous antioxidants.

\section{Conflicts of Interest}

The authors declare that there are no conflicts of interest.

\section{References}

1 Riga MG, Chelis L, Kakolyris S, et al. Transtympanic injections of Nacetylcysteine for the prevention of cisplatin-induced ototoxicity: a feasible method with promising efficacy. Am J Clin Oncol 2013; 36(01):1-6

2 Somani SM, Husain K, Jagannathan R, Rybak LP. Amelioration of cisplatin-induced oto and nephrotoxicity by protective agents. Ann Neurosci 2001;8:101-113

3 Rybak LP. Mechanisms of cisplatin ototoxicity and progress in otoprotection. Curr Opin Otolaryngol Head Neck Surg 2007;15 (05):364-369

4 McKeage MJ. Comparative adverse effect profiles of platinum drugs. Drug Saf 1995;13(04):228-244

5 Casares C, Ramírez-Camacho R, Trinidad A, Roldán A, Jorge E, García-Berrocal JR. Reactive oxygen species in apoptosis induced by cisplatin: review of physiopathological mechanisms in animal models. Eur Arch Otorhinolaryngol 2012;269(12):2455-2459

6 Dickey DT, Wu YJ, Muldoon LL, Neuwelt EA. Protection against cisplatin-induced toxicities by N-acetylcysteine and sodium thiosulfate as assessed at the molecular, cellular, and in vivo levels. J Pharmacol Exp Ther 2005;314(03):1052-1058

7 Schweitzer VG. Cisplatin-induced ototoxicity: the effect of pigmentation and inhibitory agents. Laryngoscope 1993;103(4 Pt 2):1-52

8 van Ruijven MWM, de Groot JCMJ, Klis SFL, Smoorenburg GF. The cochlear targets of cisplatin: an electrophysiological and morphological time-sequence study. Hear Res 2005a;205(1-2):241-248

9 Rybak LP, Husain K, Morris C, Whitworth C, Somani S. Effect of protective agents against cisplatin ototoxicity. Am J Otol 2000;21 (04):513-520

10 Campbell KCM, Kalkanis J, Glatz R. Ototoxicity: mechanisms, protective agents and monitoring. Curr Opin Otol Head Neck Surg 2000;8:436-440

11 Feghali JG, Liu W, Van De Water TR. L-n-acetyl-cysteine protection against cisplatin-induced auditory neuronal and hair cell toxicity. Laryngoscope 2001;111(07):1147-1155

12 Rybak LP, Mukherjea D, Jajoo S, Ramkumar V. Cisplatin ototoxicity and protection: clinical and experimental studies. Tohoku J Exp Med 2009;219(03):177-186

13 Holdiness MR. Clinical pharmacokinetics of $\mathrm{N}$-acetylcysteine. Clin Pharmacokinet 1991;20(02):123-134

14 Neuwelt EA, Pagel MA, Hasler BP, Deloughery TG, Muldoon LL. Therapeutic efficacy of aortic administration of Nacetylcysteine against bone marrow toxicity after intracarotid administration of alkylator, with or without glutathione depletion in a rat model. Cancer Res 2001;61:7868-7874

15 Strayer DS, Rubin E. Cell adaptation, cell injury and cell death. In: Rubin R, Strauer DS. Rubin's Pathology: clinicopathologic foundations of medicine, 6th edn. Philadelphia: Lippincott Williams \& Wilkins; 2012:01-46

16 Jero J, Coling DE, Lalwani AK. The use of Preyer's reflex in evaluation of hearing in mice. Acta Otolaryngol 2001;121(05):585-589

17 De Freitas MR, Figueiredo AA, Brito GAC, et al. The role of apoptosis in cisplatin-induced ototoxicity in rats. Rev Bras Otorrinolaringol (Engl Ed) 2009;75(05):745-752 
18 Hyppolito MA, Oliveira JAA, Rossato M, Holanda F. Cisplatin ototoxicity and otoprotetor to cilliated cells by ginkgo biloba extract: anatomic and eletrophisiologic study. Rev Bras Otorrinolaringol (Engl Ed) 2003;69(04):504-511

19 Hyppolito MA, de Oliveira AA, Lessa RM, Rossato M. [Amifostine otoprotection to cisplatin ototoxicity: a guinea pig study using otoacoustic emission distortion products (DPOEA) and scanning electron microscopy]. Rev Bras Otorrinolaringol (Engl Ed) 2005; 71(03):268-273

20 Gutteridge JM, Halliwell B. Antioxidants: Molecules, medicines, and myths. Biochem Biophys Res Commun 2010;393(04): 561-564

21 Rybak LP, Whitworth CA. Ototoxicity: therapeutic opportunities. Drug Discov Today 2005;10(19):1313-1321
22 Wu YJ, Muldoon LL, Neuwelt EA. The chemoprotective agent Nacetylcysteine blocks cisplatin-induced apoptosis through caspase signaling pathway. J Pharmacol Exp Ther 2005;312(02):424-431

23 Thomas Dickey D, Muldoon LL, Kraemer DF, Neuwelt EA. Protection against cisplatin-induced ototoxicity by $\mathrm{N}$-acetylcysteine in a rat model. Hear Res 2004;193(1-2):25-30

24 Muldoon LL, Pagel MA, Kroll RA, et al. Delayed administration of sodium thiosulfate in animal models reduces platinum ototoxicity without reduction of antitumor activity. Clin Cancer Res 2000;6 (01):309-315

25 Neuwelt EA, Pagel MA, Kraemer DF, Peterson DR, Muldoon LL. Bone marrow chemoprotection without compromise of chemotherapy efficacy in a rat brain tumor model. J Pharmacol Exp Ther 2004;309(02):594-599 